\title{
Why, why, why, Delilah?
}

In the case of Samson, it was the loss of his locks at the hands of his beloved that led to his downfall. For the heart under pressure, a study by Higashiyama's group finds that disaster can result from the enzyme-catalysed snipping off of growth factors that are bound to the membrane of cardiac myocytes. The paper in January's Nature Medicine pinpoints a matrix metalloprotease (MMP), ADAM12, as being a key element in the pathway that leads to cardiac hypertrophy, and often eventual heart failure.

Cardiac hypertrophy is an adaptive response to high blood pressure, and although initially protective, when prolonged, it can frequently result in sudden cardiac death. Following recent reports of the beneficial effects of MMP inhibitors during the development of congestive heart failure, attention has focused on how MMPs might contribute to the progression of heart disease. It seems that stimulation of G-protein-coupled receptors (GPCRs) by vasoactive substances such as angiotensin II leads to downstream activation of ADAM12, which cleaves membrane-bound heparin-binding epidermal growth factor-like growth factor (HB-EGF). The released HBEGF then acts on the EGF receptor to stimulate cellular growth and the development of cardiac hypertrophy. Expression of non-functional mutant variants of $A D A M 12$ in cultured cardiac myocytes almost abolished the shedding of HB-EGF in response to GPCR stimulation. Furthermore, blocking Adam 12 function in mice with the inhibitor KB-R7785 drastically reduced the hypertrophic response. However, this inhibitor was not shown to be selective for ADAM12, and, indeed, many ADAMs might be involved in the cellular remodelling that accompanies cardiac hypertophy. Although both ADAM12 and HB-EGF are potential therapeutic targets, their involvement in the initially protective response of cardiac hypertrophy means that simple inhibition of their function is unlikely to be a successful strategy.

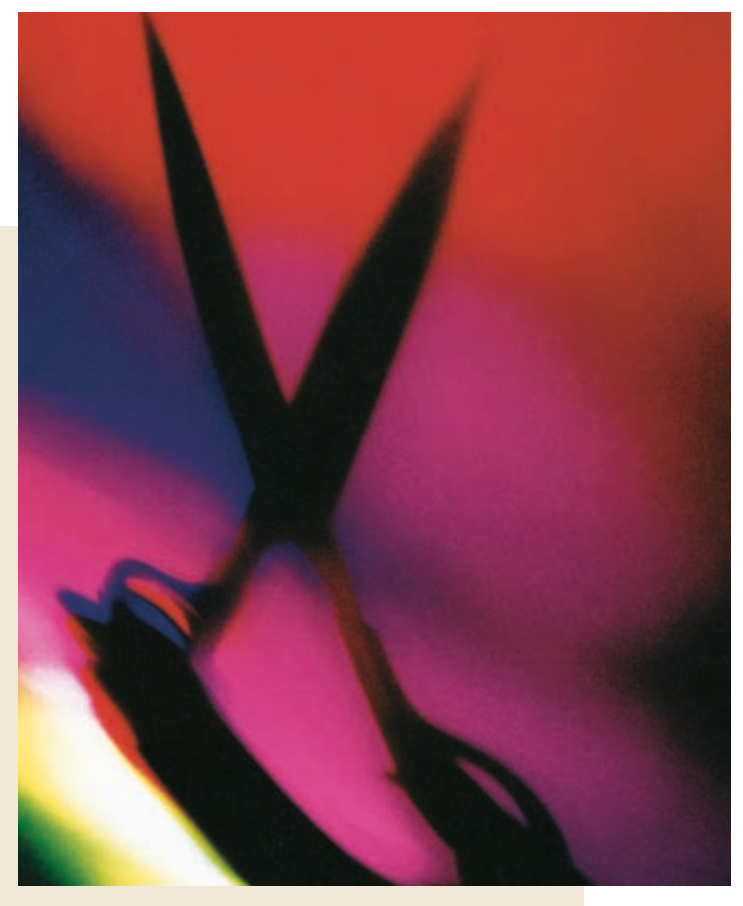

(2) References and links

Adam Smith

ORIGINAL RESEARCH PAPER Asakura, M. et al. Cardiac hypertrophy is inhibited by antagonism of ADAM12 processing of HB-EGF: metalloproteinase inhibitors as a new therapy. Nature Med. 8, 35-40 (2002)

FURTHER READING Spinale, F. G. et al. Matrix

metalloproteinase inhibition during the development of

congestive heart failure: effects on left ventricular dimensions and function. Circ. Res. 85, 364-376 (1999)

WEB SITE Higashiyama's lab:

http://www.m.ehime-u.ac.jp/school/biochem2/index.htm|

\section{Molecular beacons for DNA binding}

Transcription factors and other DNA-binding proteins are important regulators of gene expression. Many of these proteins, such as p53, are involved in the development of disease, and they are therefore attractive targets for drug development. Consequently, high-throughput methodologies for the rapid screening of DNAbinding proteins are needed. In the February issue of Nature Biotechnology, Heyduk and

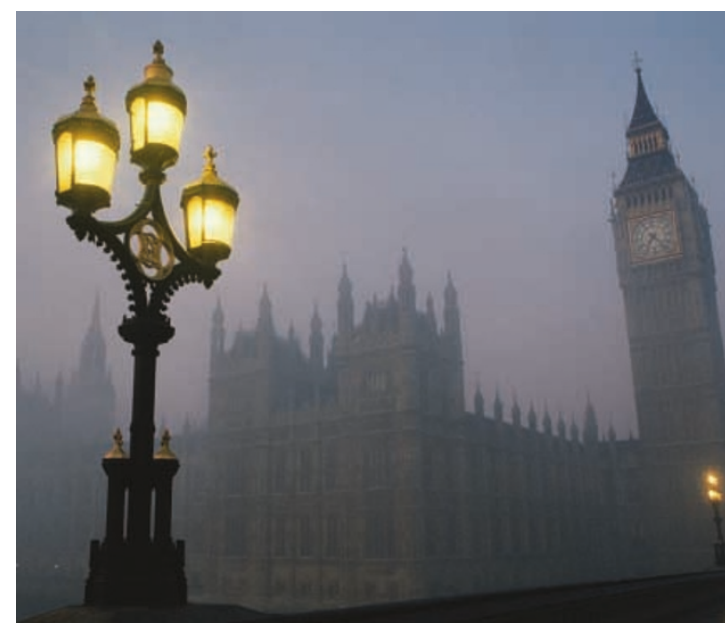

Heyduk describe a general fluorescence assay that uses DNA probes called molecular beacons to detect the sequence-specific DNA-binding activity of proteins.

The assay is based on fluorescence resonance energy transfer (FRET), which is a powerful technique for detecting the molecular proximity of fluorescently labelled molecules. The process involves the transfer of energy from a donor fluorophore in an excited state that is attached to one type of molecule, to a nearby acceptor fluorophore that is attached to another. The fluorophores are chosen so that the emission wavelength of the donor is the same as the excitation wavelength of the acceptor. Detection of fluorescent light at the emission wavelength of the acceptor molecule indicates that the donor has excited the acceptor, and that the labelled molecules are therefore less than 10-nm apart.

Although FRET is already used in protein co-localization studies, Heyduk and Heyduk have adapted this technique to detect DNA-protein binding. They designed two DNA fragments, the molecular beacons, each of which contained half of a full DNA-binding sequence labelled with either a fluorescent donor or acceptor. The efficiency of spontaneous combination of the two DNA fragments is low. However, in the presence of a suitable DNA-binding protein, the high affinity of the protein for the binding-site sequence drives the DNA fragments to combine, bringing the donor and acceptor fluorophores into close proximity, and thereby resulting in a strong FRET signal. Molecular beacons were designed and shown to work successfully for several different proteins.

Current approaches for detecting proteins with DNA-binding activity typically use radioisotopes — they are laborious and time consuming, and hard to use in high-throughput format. In addition, some proteins are hard to label with fluorescent probes. Molecular beacons allow multicolour detection of the binding activities of several proteins simultaneously, and should find wide application in high-throughput screening for drugs targeted to DNA-binding proteins, as well as in medical diagnosis and research.

(Q) References and links

Melanie Brazil

ORIGINAL RESEARCH PAPER Heyduk, T. \& Heyduk, E.

Molecular beacons for detecting DNA-binding proteins. Nature Biotechnol. 20, (in the press)

\section{WEB SITE}

Heyduk's laboratory:

http://biochemweb.slu.edu/faculty/heyduk/ 